\title{
COMPARACIÓN DE MODELOS MENSUALES Y ANUALES PARA ESTIMAR EL COEFICIENTE DE HARGREAVES EN LA COMUNIDAD VALENCIANA
}

\author{
Martí, $P^{1}(P)$, Zarzo, $M .^{2}$, Royuela, Á. ${ }^{3}$,Turégano, J.V. ${ }^{4}$ \\ 1 PDI, Universitat de les Illes Balears, Departament de Biologia, Àrea d'Enginyeria \\ Agroforestal (UIB). Ctra de Valldemossa km 7.5 07122 Palma, pau.marti@uib.cat \\ ${ }^{2}$ PDI, Departament d'Estadistica i Investigació Operativa, Universitat Politècnica de Valencia \\ (UPV). Camí de Vera s/n 46022 València, mazarcas@eio.upv.es \\ ${ }^{3}$ PDI, Universitat Politècnica de València (UPV), Centre Valencià d'Estudis sobre el Reg \\ (CVER). Camí de Vera s/n 46022 València, aroyuela@agf.upv.es \\ 4 PDI, Universitat Politècnica de València (UPV), Departament d'Enginyeria Rural i \\ Agroalimentària, Camí de Vera s/n 46022 València, jturegan@agf.upv.es
}

\section{Resumen}

El modelo de Hargreaves (HG) para estimar evapotranspiración de referencia $\left(E T_{0}\right)$ es una alternativa interesante a la ecuación de Penman Monteith (PM), propuesta como método estándar por la FAO, pero que no es aplicable en muchas situaciones porque requiere muchas variables climáticas que no suelen estar disponibles o cuyos valores medidos no son fiables. Para la aplicación de esta ecuación se recomienda una calibración local preliminar del llamado coeficiente de Hargreaves (AHC). Sin embargo, la obtención de valores concretos de $\mathrm{AHC}$ tiene un uso limitado, dado que se requieren valores locales de $\mathrm{PM}$, que se aplicará realmente para determinar la $\mathrm{ET}_{0}$ de la estación. Asimismo los valores de AHC no pueden extrapolarse. Por ello es preciso proponer y estudiar modelos para estimar el $\mathrm{AHC}$, dado que en las condiciones en las que la ecuación de HG pretende ser útil no habrá posibilidad de calibración previa mediante valores de PM.

Este estudio analiza si están justificadas las parametrizaciones mensuales o estacionales del AHC. Para ello se compararon tres escalas temporales en el desarrollo de los modelos de AHC: la anual, la mensual y la estacional. Los resultados sugieren que el desarrollo de modelos mensuales podría reducir el error relativo alrededor del $2 \%$ para $\mathrm{AHC}$ diarios y alrededor de $1 \%$ para AHC medios mensuales, es decir, un único modelo anual de AHC podría ser insuficiente para recoger toda la variabilidad anual de AHC. Por ello, la aplicación de modelos mensuales (o estacionales) podría estar justificado para una correcta estimación de los AHC. Asimismo, los resultados muestran que la estimación mensual del AHC fue más ajustada de mayo a septiembre que de octubre a abril, y, particularmente, que de noviembre a enero.

\section{1- Introducción}

La estimación precisa de la evapotranspiración (ET) puede contribuir a mejorar el diseño y gestión de instalaciones de riego. La ET es un parámetro crucial del ciclo hidrológico en agricultura, particularmente en sistemas de regadío. Jensen (1968) introdujo la metodología ampliamente extendida de estimar la ET como el producto de la evapotranspiración de referencia $\left(\mathrm{ET}_{\mathrm{o}}\right)$, i.e. la $\mathrm{ET}$ de una superficie de referencia, y un coeficiente de cultivo, representativo de prácticas culturales, tipo de cultivo y desarrollo del mismo. 
La ecuación de Penman-Monteith (FAO56 PM), (Allen et al. 1998), es el método de referencia propuesto por la $\mathrm{FAO}$ para determinar la $\mathrm{ET}_{0} \mathrm{y}$ validar otras ecuaciones, pero en muchas situaciones no es aplicable porque requiere muchas variables climáticas (temperatura, humedad relativa, radiación solar y velocidad del viento) que no suelen estar disponibles o cuya medida puede no ser fiable. Este hecho ha dado lugar al desarrollo de ecuaciones más sencillas que requieren menos variables climáticas de entrada. La

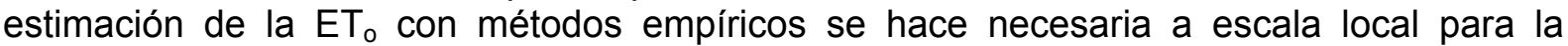
gestión de recursos hídricos y del riego en particular, debido a que no es posible obtener valores experimentales de $\mathrm{ET}_{\mathrm{o}} \mathrm{o}$ aplicar otros modelos más complejos.

Sin embargo, la validez de muchos de estos modelos empíricos depende de los rangos climáticos para los que fueron calibrados. Por otra parte, la aplicación de la ecuación FAO56 PM adoptando valores estimados de los inputs en lugar de valores medidos puede acarrear también errores. El estudio de modelos térmicos de $\mathrm{ET}_{0}$ está justificado por diversas razones. En primer lugar, la temperatura y la radiación solar explican al menos el $80 \%$ de la variabilidad de la $\mathrm{ET}_{\text {。 }}$ (Samani 2000). En segundo lugar, diversos estudios indican que el rango térmico diario puede relacionarse con la humedad relativa y la nubosidad (Di Stefano and Ferro 1997). En tercer lugar, la advección depende de la interacción entre temperatura, humedad relativa, presión de vapor y velocidad del viento, y estas variables pueden relacionarse con el rango térmico (Vanderlinden et al. 2004). Finalmente, la temperatura constituye la variable más ampliamente disponible en las estaciones climáticas de entre las que se necesitan para el cálculo de la ET。 (Mendicino and Senatore 2013).

La ecuación de Hargreaves, HG, (Hargreaves y Samani 1985) sólo requiere datos medidos de temperatura media del aire y rango térmico, al margen de valores calculados de radiación extraterrestre. Jensen et al. (1997) recomendó la ecuación de HG como una de las más simples y exactas de entre las ecuaciones empíricas para estimar $\mathrm{ET}_{\mathrm{o}}$. Según Allen et al. (1998), la ecuación de HG proporciona estimaciones razonables de $\mathrm{ET}_{0}$ con validez global. A pesar de que se han constatado aplicaciones de HG a escala diaria con bajos errores (Di Stefano and Ferro 1997), Hargreaves y Allen (2003) sugirieron que las estimaciones más precisas serían de esperar a partir de periodos de cinco días o superiores, debido a que las estimaciones diarias están sujetas a una mayor variabilidad, causada por el movimiento de frentes, la fluctuación de la velocidad del viento y la nubosidad. Sin embargo, numerosas aplicaciones agrícolas e hidrológicas requieren datos diarios de $\mathrm{ET}_{\mathrm{o}}$.

Según Maestre-Valero et al. (2013), la validez de la ecuación original de HG se ve fuertemente influenciada por las condiciones climáticas locales bajo las que se calibró. Según Samani (2000), la ecuación de HG no debería extrapolarse sin ser previamente calibrada a escala local. Esta calibración puede llevarse a cabo usando valores

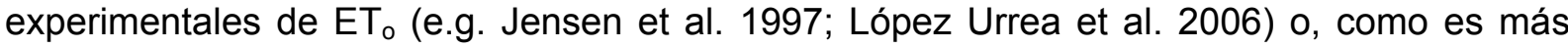
habitual en la práctica, mediante valores calculados de la ecuación FAO56 PM (e.g. Vanderlinden et al. 2004; Gavilán et al. 2006; Mendicino y Senatore 2013). El proceso de calibración da lugar a valores ajustados del coeficiente de Hargreaves (AHC).

Sin embargo, las ecuaciones calibradas de HG tienen sólo validez local y no pueden ser extrapoladas a otros lugares donde no existen valores de referencia de $\mathrm{ET}_{\text {}}$ para efectuar una calibración local. De hecho, en las estaciones donde se puede calibrar la ecuación de HG porque hay variables suficientes para calcular los valores de referencia requeridos de $\mathrm{ET}_{\text {。 }} \mathrm{FAO56} \mathrm{PM}$, se utilizaría directamente ésta última como método para calcular la $\mathrm{ET}_{0}$, dejando relegado el método de HG para casos de fallo del sistema de medida. Por ello, además de la calibración local de los AHCs, diferentes autores han sugerido la calibración paramétrica de AHC, es decir, la modelización de AHC a partir de diferentes inputs, como rango térmico (Samani 2000; Mendicino y Senatore 2013; Maestre-Valero et al. 2013), el cociente temperatura media-rango térmico $\left(\mathrm{T}_{\text {media }} / \Delta \mathrm{T}\right)$ (Vanderlinden et al. 2004; Lee 2010; 
Thepadia y Martínez 2012; Mendicino y Senatore 2013; Maestre-Valero et al. 2013; Martí et al. 2015), velocidad del viento (Jensen et al. 1997; Martínez-Cob y Tejero-Juste 2004), humedad relativa (Hargreaves y Allen 2003), precipitación (Droogers y Allen 2002), y altitud (Ravazzani et al. 2012). Shahidian et al. (2013) llevaron a cabo un análisis en profundidad de los siete parámetros más prometedores para efectuar la calibración de HG testando diferentes modelos bajo condiciones climáticas uniformes y no uniformes. Como conclusión, sugirieron que la velocidad del viento aparecía como el parámetro más importante para mejorar las estimaciones de HG. Sin embargo, los valores de velocidad del viento no suelen estar disponibles en los casos en los que la ecuación de HG puede ser útil.

Hasta hoy los estudios sobre modelización del AHC han propuesto fundamentalmente modelos que calculan un único valor de $\mathrm{AHC}$ por estación, excepto en algún caso donde se han propuesto modelos mensuales (e.g. Maestre-Valero et al. 2013). Sin embargo, no se han encontrado análisis comparativos entre ambos procedimientos. El trabajo actual pretende evaluar si la obtención de modelos mensuales de AHC podría estar justificada frente a la metodología de obtener un único AHC por estación. Para ello se compararán modelos mensuales y anuales de AHC en 30 estaciones de la Comunidad Valenciana.

\section{2- Métodos}

\subsection{Datos considerados}

En este estudio se han utilizado datos diarios de temperatura máxima ( $\left.T_{\max }\right)$, mínima $\left(T_{\min }\right)$ y media $\left(T_{\mathrm{m}}\right)$ del aire, humedad relativa $(\mathrm{HR})$, radiación solar $\left(R_{\mathrm{s}}\right)$ y velocidad del viento a $2 \mathrm{~m}$ de altura $\left(u_{2}\right)$ obtenidos en 30 estaciones de la Comunidad Valenciana durante el periodo 2000-2007. Los datos fueron suministrados por el Servicio de Tecnología del Riego del Instituto Valenciano de Investigaciones Agrarias (http://estaciones.ivia.es). Para obtener más información sobre las estaciones y las condiciones climáticas del área de estudio puede consultarse Martí y Zarzo (2012).

\subsection{Métodos para estimar $\mathrm{ET}_{\mathrm{o}}$}

\subsubsection{Ecuación FAO56 Penman Monteith}

Debido a la ausencia de lisímetro $u$ otros instrumentos para medir experimentalmente la ET, se aplicó la ecuación FAO56-PM para validar las estimaciones y para calibrar la ecuación de HG. Esta práctica es muy habitual en ausencia de datos experimentales y está

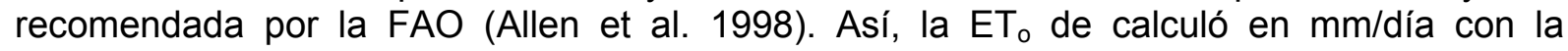
expresión

$$
E T_{o}^{P M}=\frac{0.408 \Delta\left(R_{n}-G\right)+\gamma \frac{900}{T+273} u_{2}\left(e_{s}-e_{a}\right)}{\Delta+\gamma\left(1+0.34 u_{2}\right)},
$$

donde $R_{\mathrm{n}}$ es la radiación neta en la superficie del cultivo $\left(\mathrm{MJ} / \mathrm{m}^{2} /\right.$ día); $G$ es la densidad de flujo de calor del suelo ( $\mathrm{MJ} / \mathrm{m}^{2} /$ día); $T$ es la temperatura diaria media del aire a $2 \mathrm{~m}$ de altura $\left({ }^{\circ} \mathrm{C}\right) ; \gamma$ es la constante psicrométrica $\left(\mathrm{kPa} /{ }^{\circ} \mathrm{C}\right) ; \Delta$ es la pendiente de la curva de presión de vapor $\left(\mathrm{kPa} /{ }^{\circ} \mathrm{C}\right)$; $e_{\mathrm{s}}$ es la presión de saturación de vapor $(\mathrm{kPa}) ; e_{\mathrm{a}}$ es la presión de vapor real $(\mathrm{kPa}) ; \mathrm{y} u_{2}$ es la velocidad del viento a $2 \mathrm{~m}$ de altura $(\mathrm{m} / \mathrm{s})$. Todos los parámetros se calcularon según las ecuaciones propuestas por Allen et al. (1998). G se supuso 0 para cálculos diarios, mientras que para cálculos mensuales se aplició la expresión

$$
G_{\text {mes } i}=0.07\left(T_{\text {mes } i+1}-T_{\text {mes } i-1}\right)
$$


donde $G_{\text {mes } i}$ es el flujo de calor del suelo en el mes $i, T_{\text {mes } i+1}$ es la temperatura media en el mes $i+1$, mientras que $T_{\text {mes } i-1}$ es la temperatura media en el mes $i-1$.

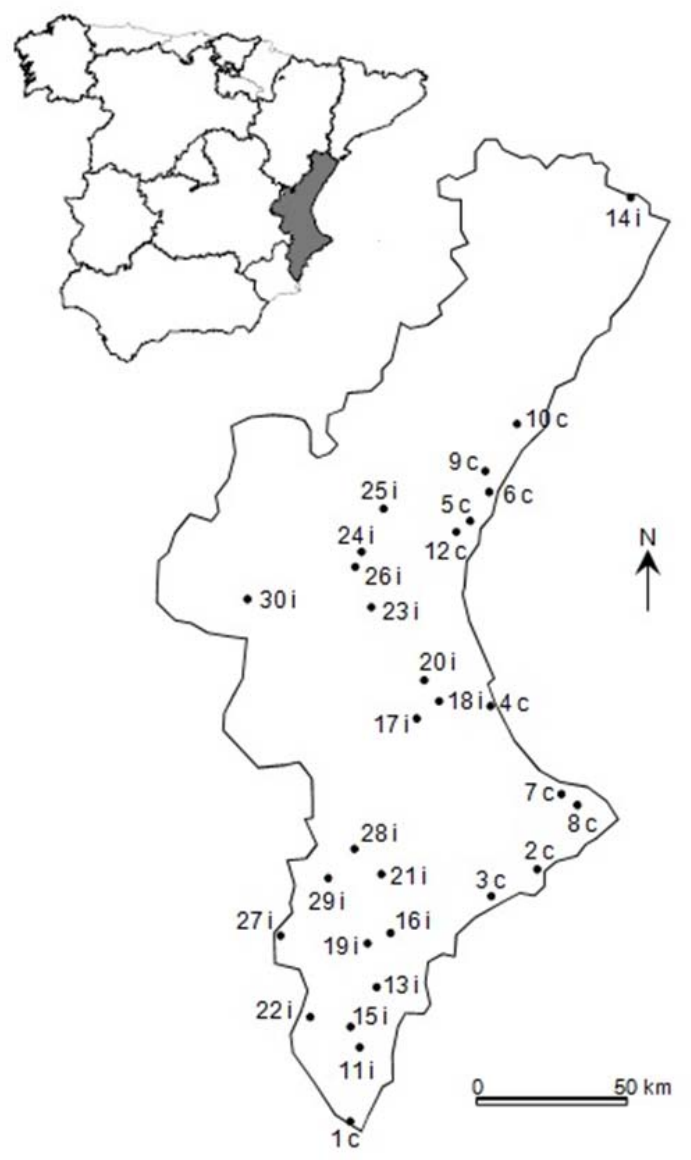

Figura 1. Localización de las estaciones estudiadas (Martí et al. 2015). 'c' indica estación costera, 'i' indica estación interior

\subsubsection{Ecuación de Hargreaves}

La ecuación de HG para estimar evapotranspiración de referencia $\left(E T_{0}{ }^{H G}, \mathrm{~mm} / \mathrm{día}\right)$ es según Hargreaves y Samani (1985)

$$
E T_{o}^{H G}=A H C R_{a}(T+17.8) \sqrt{\Delta T}
$$

donde $R_{\mathrm{a}}$ es el equivalente en $\mathrm{mm}$ de agua de la radiación extraterrestre ( $\mathrm{mm} /$ día); $\Delta T$ es el rango térmico diario $\left({ }^{\circ} \mathrm{C}\right) ; T$ es la temperatura media diaria $\left({ }^{\circ} \mathrm{C}\right), A H C$ es el coeficiente ajustado de Hargreaves, igual a 0.0023 en la ecuación original.

La evolución histórica de la ecuación de HG puede encontrarse en Hargreaves y Allen (2003), donde se detalla la evolución de los valores de AHC. Vanderlinden et al. (2004) sugiere que $A H C$ parece aumentar en áreas costeras, donde $\Delta T$ disminuye debido al efecto del mar, y parece disminuir en zonas montañosas, donde el movimiento de las masas de aire eleva el rango térmico. Vanderlinden et al. (2004) propusieron la siguiente expresión para estimar AHC basándose en el análisis de 16 estaciones en Andalucía durante un periodo de 38 años 


$$
A H C=k_{1} \frac{T_{m}}{\Delta T}+k_{2}
$$

donde $T_{\mathrm{m}}$ y $\Delta T$ corresponden a la temperatura media y al rango térmico medio por estación. Concretamente, propusieron los valores $k_{1}=0.0005$ y $k_{2}=0.00159$, con una bondad de ajuste de $R^{2}=0.90$. La misma expresión fue reajustada por Lee (2010) en la península de Corea con datos de 21 estaciones durante 10 años, y obtuvo $k_{1}=0.0004$ y $k_{2}=0.0013\left(R^{2}=0.84\right)$. La misma ecuación fue calibrada por Thepadia y Martínez (2012) usando datos de 22 estaciones en Florida durante 14 años, llegando a los valores $k_{1}=0.000411$ and $k_{2}=0.00132$ $\left(R^{2}=0.97\right)$. Mendicino y Senatore (2013) recalibraron la misma expresión en el sur de Italia con datos de 137 estaciones y obtuvieron $k_{1}=0.0006$ y $k_{2}=0.00121\left(R^{2}=0.46\right)$ considerando todas las estaciones, y $k_{1}=0.0006$ and $k_{2}=0.00097\left(R^{2}=0.83\right)$ considerando sólo estaciones costeras. Asimismo, adaptaron el modelo de Samani, basado en la estimación indirecta de $\mathrm{AHC}(A H C=0.0135 \cdot C)$ a partir de rango térmico:

$$
A H C=1.23057 \cdot 10^{-5} \Delta T^{2}-3.9237 \cdot 10^{-4} \Delta T+4.80226 \cdot 10^{-3}\left(\mathrm{R}^{2}=0.77\right)
$$

A partir de la expresión del coeficiente de Samani recalibrado

$$
C=0.0009115 \Delta T^{2}-0.02906 \Delta T+0.3557
$$

Finalmente, Martí et al. (2015) testaron los modelos de Vanderlinden et al. (2004) y Samani (2000), respectivamente, en la Comunidad Valenciana y constataron que no proporcionaban estimaciones suficientemente ajustadas del AHC, por lo que propusieron nuevos modelos incluyendo nuevas variables de entrada. La exactitud de las estimaciones de AHC se pudo incrementar, por una parte, incluyendo en los modelos datos geográficos de las estaciones (10), y, por otra, incluyendo información cualitativa sobre el rango de velocidad del viento (11).

$$
\begin{gathered}
\mathrm{AHC}=0.003704-0.000149 \cdot \Delta \mathrm{T}+0.000261 \cdot \tau+7.5212 \cdot 10^{-7} \cdot \mathrm{z} \\
\mathrm{AHC}=0.003456-0.000127 \cdot \Delta \mathrm{T}+0.000174 \cdot \tau+5.8737 \cdot 10^{-7} \cdot \mathrm{z}+0.000152 \cdot \mathrm{u}_{0-1}
\end{gathered}
$$

donde $\Delta T$ es el rango térmico, $\tau$ es la longitud, $z$ es la altitud y $u_{0-1}$ es la clase cualitativa de velocidad del viento.

\subsection{Desarrollo de los modelos}

En este estudio se van a comparar dos de las combinaciones de inputs sugeridas por Martí et al. (2015) con la particularidad de que se calibrarán a tres escalas temporales diferentes: la anual, la estacional y la mensual. En el primer caso se obtiene un único modelo de $\mathrm{AHC}$, en el segundo caso se obtienen 3 modelos, uno para verano, otro para invierno y otro para el resto del año, mientras que en el tercer caso se obtienen 12 modelos de $A H C$, uno por mes.

Los valores diarios de $\mathrm{AHC}$ se obtuvieron multiplicando por 0.0023 el ratio de $\mathrm{ET}_{0}{ }^{\mathrm{PM}}$ entre $\mathrm{ET}_{0}{ }^{\mathrm{HG}}$. Estos valores 'observados' de AHC sirvieron para obtener los modelos aplicando regresión lineal múltiple (RLM) a partir de inputs diarios (escala diaria). En el primer caso se consideró una única matriz global. En el segundo caso, los modelos se obtuvieron aplicando RLM a los datos de cada estación (3 estaciones). Finalmente, en el tercer caso, los modelos se obtuvieron individualmente por mes, también a escala diaria (e.g. el modelo para enero se obtuvo con todos los valores diarios de enero de todas las estaciones). Asimismo, la obtención de los modelos anteriores se repitió considerando esta 
vez valores medios mensuales de $A H C$ y del resto de variables climáticas involucradas (escala mensual), obtenidos del promedio de los datos diarios de cada mes en cada estación, y de la aplicación de la ecuación de $\mathrm{ET}_{\text {o }}$ para el intervalo mensual según Allen et al. (1998).

Además, como se ha comentado, se consideraron dos combinaciones de inputs a partir de los resultados de Martí et al. (2015): a) modelo 1, que considera las variables de partida

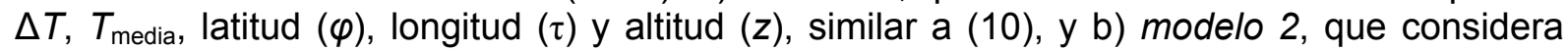
como inputs de partida los de a) y $u_{0-1}$ (velocidad cualitativa del viento considerando dos clases, muy ventoso/poco ventoso), similar a (11). Los inputs significativos para en cada caso podrían variar al aplicar RLM. Dichos modelos RLM se obtuvieron aplicando el software Statgraphics plus 5.1 (StatPoint Technologies Inc., Warrenton, VA, USA). EI resto de cálculos se implementaron con Matlab.

\subsection{Evaluación de los modelos}

La exactitud de las estimaciones se evaluó mediante la raíz cuadrada del error cuadrático medio en valor relativo (RRMSE),

$$
\text { RRMSE }=\frac{1}{\bar{x}} \cdot \sqrt{\frac{1}{n} \sum_{i=1}^{n}\left(x_{i}-\hat{x}_{i}\right)^{2}}
$$

y el error medio absoluto (MAE)

$$
M A E=\frac{1}{n} \cdot \sum_{i=1}^{n}\left|x_{i}-\hat{x}_{i}\right|
$$

donde $n$ y $\hat{x}_{i}$ son el número total de observaciones y los valores de $\mathrm{ET}_{0}$ calculados, respectivamente, $x_{i}$ son los valores de referencia, y $\bar{x}$ es el valor medio de los valores de referencia.

\section{3- Resultados y Discusión}

En la Tabla 1 se presentan los valores medios de AHC obtenidos por mes y estación, promediados a partir de los valores diarios calculados. Aunque el objetivo del estudio va más encaminado a evaluar los modelos de estimación de AHC, estos valores también tienen valor para ser aplicados en las estaciones estudiadas por mes o globalmente. Dicha tabla revela que las oscilaciones del valor de AHC pueden llegar a ser relevantes dentro del ciclo anual. Los rangos anuales medios de AHC oscilan entre 0.00014 (0.00206-0.00192, estación 25) y 0.00098 (0.00341-0.00243, estación 27).

La Tabla 2 muestra los indicadores medios de los modelos 1 y 2 en todas las estaciones usando AHC diarios y AHC medios mensuales. Estos resultados revelan que la consideración de un modelo de AHC para cada mes permite reducir el error relativo del modelo 1 (temperatura e información geográfica como inputs) alrededor de 0.02 (0.1092 vs. $0.1257)$ a escala diaria y alrededor de 0.01 (0.0519 vs. 0.0633) a escala media mensual. Los modelos estacionales sólo mejoran muy levemente el error medio de los modelos anuales ( 0.1225 vs. 0.1257 y 0.0504 vs. 0.0633 ). Las mismas pautas cualitativas se mantienen al comparar las escalas mensual, estacional y anual del modelo 2 (que incluye, además, velocidad cualitativa del viento).

Finalmente, como era de esperar atendiendo a los resultados ya presentados por Martí et al. (2015), los índices del modelo 2 mejoran respecto de los del modelo 1 , 
precisamente por incluir información cualitativa sobre el régimen de velocidad del viento local. La reducción de los rangos de los errores en los modelos que consideran AHC medios mensuales se explica por la reducción de la variabilidad introducida al promediar las variables. Sin embargo, cuando se calcule la $\mathrm{ET}_{0}$, los modelos de escala diaria considerarán un AHC estimado diferente para cada día, mientras que los modelos de la escala mensual asignarán el mismo AHC estimado para todos los días del mismo mes.

Tabla 1. Valores mensuales medios de AHC en las estaciones estudiadas.

\begin{tabular}{|c|c|c|c|c|c|c|c|c|c|c|c|c|}
\hline \multirow{2}{*}{ e } & \multicolumn{12}{|c|}{ mes } \\
\hline & 1 & 2 & 3 & 4 & 5 & 6 & 7 & 8 & 9 & 10 & 11 & 12 \\
\hline 1 & 0.00288 & 0.00262 & .00254 & 0.00250 & 0.00249 & 0.00257 & 0.00262 & 0.00261 & 0.00263 & 0.00264 & 0.00293 & 0.00304 \\
\hline 2 & 0.00237 & 0.00227 & 0.00230 & 0.00236 & 0.00233 & 0.00238 & 0.00250 & 0.00250 & 0.00250 & 0.00231 & 0.00252 & 0.00248 \\
\hline 3 & 00273 & 0.00245 & .00234 & 0.00238 & 0.00233 & 0.00236 & 0.00246 & 0.00251 & 0.00254 & 0.00257 & 0.00279 & 0.00279 \\
\hline 4 & 0.00257 & 0.00240 & 0.00234 & 0.00230 & 0.00229 & 0.00238 & 0.00251 & 0.00246 & 0.00239 & 0.00220 & 0.00276 & 0.00264 \\
\hline 5 & 0.00251 & 0.00238 & 0.00228 & 0.00231 & 0.00229 & 0.00232 & 0.00249 & 0.00245 & 0.00252 & 0.00240 & 0.00269 & 0.00271 \\
\hline 6 & 0.00194 & 0.00190 & 0.00190 & 0.00196 & 0.00195 & 0.00199 & 0.00210 & 0.00207 & 0.00209 & 0.00201 & 0.00212 & 0.00209 \\
\hline 7 & 0.00195 & 0.00192 & 0.00192 & 0.00197 & 0.00191 & 0.00198 & 0.00206 & 0.00203 & 0.00199 & 0.00195 & 0.00211 & 0.00205 \\
\hline 8 & 0.00177 & 0.00174 & 0.00179 & 0.00188 & 0.00186 & 0.00191 & 0.00199 & 0.00197 & 0.00193 & 0.00185 & 0.00190 & 0.00183 \\
\hline 9 & 0.00254 & 0.00235 & 0.00226 & 0.00229 & 0.00221 & 0.00225 & 0.00234 & 0.00231 & 0.00233 & 0.00238 & 0.00263 & 0.00272 \\
\hline 10 & 0.00207 & 0.00200 & 0.00197 & 0.00210 & 0.00204 & 0.00213 & 0.00225 & 0.00225 & 0.00223 & 0.00212 & 0.00225 & 0.00209 \\
\hline 11 & 00258 & 0.00244 & 0.00239 & 0.00236 & 0.00233 & 0.00237 & 0.00247 & 0.00246 & 0.00241 & 0.00241 & 0.00269 & 0.00272 \\
\hline 12 & 00200 & 0.00201 & 0.00192 & 0.00201 & 0.00195 & 0.00202 & 0.00213 & 0.00206 & 0.00203 & 0.00199 & 0.00202 & 0.00189 \\
\hline 13 & 0.00205 & 0.00200 & 0.00205 & 0.00211 & 0.00209 & 0.00218 & 0.00225 & 0.00225 & 0.00223 & 0.00210 & 0.00226 & 0.00213 \\
\hline 14 & 00278 & 0.00263 & .00241 & 0.00240 & 0.00232 & 0.00226 & 0.00242 & 0.00243 & 0.00245 & 0.00241 & 0.00284 & 0.00289 \\
\hline 15 & 0.00201 & 0.00199 & 0.00198 & 0.00197 & 0.00195 & 0.00200 & 0.00205 & 0.00200 & 0.00204 & 0.00201 & 0.00218 & 0.00212 \\
\hline 16 & 0.00293 & 0.00267 & 0.00259 & 0.00248 & 0.00237 & 0.00237 & 0.00242 & 0.00246 & 0.00246 & 0.00253 & 0.00279 & 0.00294 \\
\hline 17 & 0.00164 & 0.00171 & 0.00181 & 0.00181 & 0.00183 & 0.00187 & 0.00201 & 0.00199 & 0.00197 & 0.00186 & 0.00186 & 0.00174 \\
\hline 18 & 00159 & 0.00162 & 0.00171 & 0.00180 & 0.00176 & 0.00188 & 0.00197 & 0.00196 & 0.00189 & 0.00178 & 0.00173 & 0.00158 \\
\hline 19 & 0.00284 & 0.00251 & .00240 & 0.00230 & 0.00216 & 0.00216 & 0.00220 & 0.00217 & 0.00218 & 0.00227 & 0.00265 & 0.00288 \\
\hline 20 & 0.00220 & 0.00222 & 0.00216 & 0.00207 & 0.00194 & 0.00195 & 0.00206 & 0.00206 & 0.00202 & 0.00204 & 0.00224 & 0.00225 \\
\hline 21 & 0.00295 & 0.00288 & 0.00275 & 0.00259 & 0.00245 & 0.00254 & 0.00263 & 0.00259 & 0.00259 & 0.00262 & 0.00295 & 0.00300 \\
\hline 22 & 0.00235 & 0.00235 & 0.00234 & 0.00226 & 0.00220 & 0.00229 & 0.00235 & 0.00231 & 0.00230 & 0.00222 & 0.00232 & 0.00242 \\
\hline 23 & 0.00276 & 0.00253 & 0.00237 & 0.00223 & 0.00202 & 0.00193 & 0.00199 & 0.00202 & 0.00205 & 0.00222 & 0.00257 & 0.00275 \\
\hline 24 & 0.00272 & 0.00256 & 0.00239 & 0.00233 & 0.00212 & 0.00215 & 0.00222 & 0.00222 & 0.00225 & 0.00232 & 0.00274 & 0.00278 \\
\hline 25 & 0.00201 & 0.00197 & 0.00199 & 0.00200 & 0.00192 & 0.00197 & 0.00205 & 0.00205 & 0.00204 & 0.00196 & 0.00206 & 0.00194 \\
\hline 26 & 0.00228 & 0.00217 & 0.00209 & 0.00204 & 0.00185 & 0.00180 & 0.00183 & 0.00183 & 0.00190 & 0.00206 & 0.00234 & 0.00232 \\
\hline 27 & 0.00341 & 0.00303 & 0.00284 & 0.00264 & 0.00243 & 0.00245 & 0.00247 & 0.00250 & 0.00255 & 0.00273 & 0.00322 & 0.00341 \\
\hline 28 & 0.00251 & 0.00242 & 0.00242 & 0.00234 & 0.00223 & 0.00231 & 0.00238 & 0.00242 & 0.00247 & 0.00249 & 0.00258 & 0.00259 \\
\hline 29 & 0.00245 & 0.00243 & 0.00240 & 0.00228 & 0.00218 & 0.00221 & 0.00227 & 0.00225 & 0.00229 & 0.00225 & 0.00240 & 0.00248 \\
\hline 30 & 0.00239 & 0.00239 & 0.00236 & 0.00226 & 0.00211 & 0.00210 & 0.00216 & 0.00222 & 0.00225 & 0.00228 & 0.00243 & 0.00240 \\
\hline
\end{tabular}

La figura 2 presenta los valores desglosados del RRMSE para los modelos 1 y 2 considerando el intervalo mensual y la escala diaria de AHC. Se comprueba que la estimación de los AHC presenta errores relativos considerablemente menores de mayo a septiembre que en el resto de meses, especialmente en invierno (noviembre a febrero). Ello podría vincularse a una menor variabilidad de los parámetros climáticos durante ese periodo. 
El desglose del error anual en errores mensuales, i.e. utilizando modelos anuales, presenta un patrón similar (resultados no mostrados). Asimismo, las diferencias de RRMSE entre los modelos 1 y 2 parecen reducirse también entre mayo y septiembre, lo que podría indicar que los datos de temperatura tendrían un nivel de significación más alto que los de velocidad cualitativa del viento durante este periodo en la estimación de $\mathrm{AHC}_{\mathrm{H}} \mathrm{ET}_{\mathrm{o}}$.

Tabla 2. Indicadores de los modelos de AHC para las escalas temporales consideradas.

\begin{tabular}{|c|c|c|c|c|}
\hline \multirow[b]{2}{*}{ intervalo } & \multirow[b]{2}{*}{ modelo } & \multicolumn{3}{|c|}{$\mathrm{AHC}$} \\
\hline & & $\begin{array}{c}\text { RRMSE } \\
(-)\end{array}$ & $\begin{array}{l}R^{2} \\
(-)\end{array}$ & $\begin{array}{c}\text { MAE } \\
(-)\end{array}$ \\
\hline \multicolumn{5}{|c|}{ escala diaria } \\
\hline \multirow{2}{*}{ mes } & 1 & 0.1092 & 0.570 & 0.000177 \\
\hline & 2 & 0.0895 & 0.711 & 0.000145 \\
\hline \multirow{2}{*}{ estación } & 1 & 0.1225 & 0.459 & 0.000204 \\
\hline & 2 & 0.0972 & 0.659 & 0.000156 \\
\hline \multirow{2}{*}{ año } & 1 & 0.1257 & 0.431 & 0.000207 \\
\hline & 2 & 0.1063 & 0.592 & 0.000174 \\
\hline \multicolumn{5}{|c|}{ escala mensual } \\
\hline \multirow{2}{*}{ mes } & 1 & 0.0519 & 0.853 & 0.0000885 \\
\hline & 2 & 0.0416 & 0.905 & 0.0000699 \\
\hline \multirow{2}{*}{ estación } & 1 & 0.0504 & 0.861 & 0.0000859 \\
\hline & 2 & 0.0425 & 0.901 & 0.0000711 \\
\hline \multirow{2}{*}{ año } & 1 & 0.0633 & 0.782 & 0.000112 \\
\hline & 2 & 0.0551 & 0.834 & 0.0000976 \\
\hline
\end{tabular}




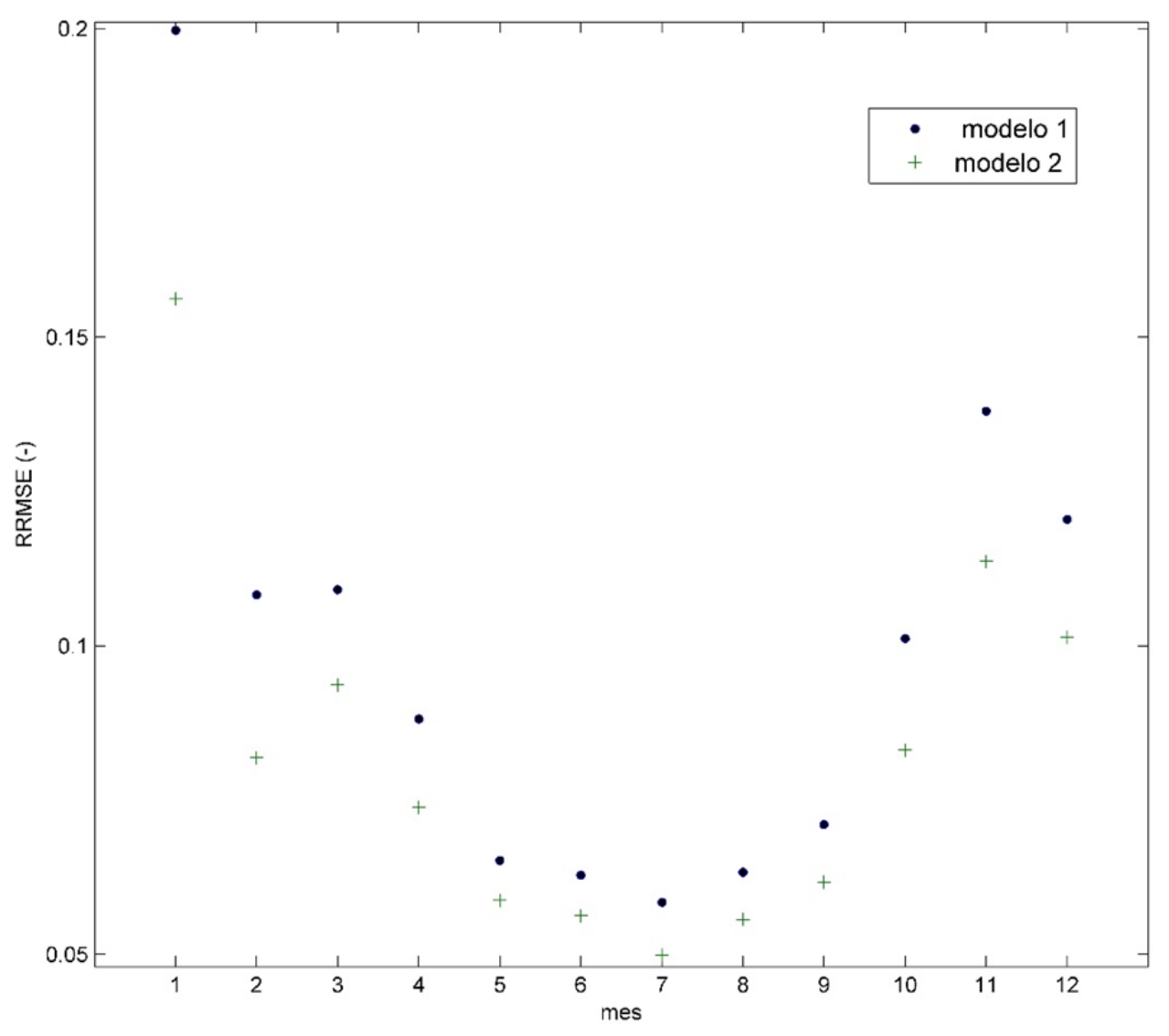

Figura 2. Valores del RRMSE medio por mes de los 12 modelos mensuales 1 y 2 en las 30 estaciones para el caso de escala diaria de AHC.

\section{4- Conclusiones}

Este trabajo presenta un análisis comparativo de modelos para estimar el coeficiente ajustado de Hargreaves considerando diferentes criterios temporales para definir los datos de calibración. Concretamente, se compararon tres escalas temporales en el desglose del set de datos para calibrar el modelo: la anual, la mensual y la estacional. Además, las tres escalas anteriores se aplicaron en modelos para estimar valores diarios de AHC y en modelos para estimar valores medios mensuales de AHC. $Y$ todo ello para dos combinaciones de inputs: una basada en datos geográficos y térmicos, y otra que incluía, además, velocidad cualitativa del viento (poco ventoso, muy ventoso).

Los resultados sugieren que el desarrollo de modelos mensuales podría reducir el error relativo alrededor de un $2 \%$ para valores diarios de $\mathrm{AHC}$ y alrededor de un $1 \%$ para valores medios mensuales de $\mathrm{AHC}$, es decir, la aplicación de un solo modelo anual para estimar un único valor de AHC por estación podría ser insuficiente para recoger toda la variabilidad anual de AHC. Por ello, la aplicación de diferentes modelos mensuales (o estacionales) podría estar justificado para una correcta estimación de los AHC.

El desglose por mes de los indicadores de los AHC sugiere que la estimación de mayo a septiembre es más precisa que de octubre a abril, y particularmente, que de noviembre a enero. Esto se produce tanto para la estimación de los AHC a partir de modelos mensuales como para el desglose por mes de las estimaciones de los modelos anuales. Ello podría deberse a una menor variabilidad de los parámetros climáticos, incluyendo la $\mathrm{ET}_{\circ}$ y el $\mathrm{AHC}$, durante estos meses.

El estudio de modelos paramétricos de $\mathrm{AHC}$ es de especial relevancia para la aplicación de la ecuación de Hargreaves. Ello se debe, por una parte, a que se recomienda 
calibrar la ecuación original antes de ser aplicada localmente, y, por otra parte, a que dicha calibración no podrá efectuarse a partir de valores de referencia de la ecuación FAO56 PM, ya que sólo habría valores medidos de temperaturas disponibles y/o fiables. La única excepción sería una situación de fallo del sistema de medida. En este caso, además de valores de temperatura, también habría valores históricos locales de FAO56 PM, que permitirían calibrar la ecuación de Hargreaves con valores locales de AHC.

\section{5- Bibliografía}

Allen, R.G., Pereira, L.S., Raes, D., Smith, M. (1998). Crop evapotranspiration. Guidelines for computing water requirements. FAO Irrigation and Drainage, Paper 56. FAO, Rome.

Di Stefano, C., Ferro, V. (1997). Estimation of evapotranspiration by Hargreaves formula and remote sensed data in semi-arid Mediterranean areas. J. Agric. Eng. Res., 68, 189199.

Droogers, P. \& Allen, R.G. (2002). Estimating reference evapotranspiration under inaccurate data conditions. Irrig. Drain. Syst., 16(1), 33-45.

Gavilán, P., Lorite, I.J., Tornero, S., Berengena, J. (2006). Regional calibration of Hargreaves equation for estimating reference ET in a semi arid environment. Agric. Water Manage., 81, 257-281.

Hargreaves, G.H. \& Allen, R.G. (2003). History and evaluation of Hargreaves evapotranspiration equation. J. Irrig. Drain. Eng., 129(1), 53-63.

Hargreaves, G.H., Samani, Z.A. (1985). Reference crop evapotranspiration from ambient air temperature. Appl. Eng. Agric. 1 (2), 96-99.

Jensen, D.T., Hargreaves, G.H., Temesgen, B., Allen, R.G. (1997). Computation of ET。 under non ideal conditions. J. Irrig. Drain. Eng, 123(5), 394-400.

Lee, K.H. (2010). Relative comparison of the local recalibration of the temperature-based equation for the Korea Peninsula. J. Irrig. Drain. Eng., 136(9), 585-594.

López-Urrea, R., Martín de Santa Olalla, F., Fabeiro, C., Moratalla, A. (2006) Testing evapotranspiration equations using lysimeter observations in a semiarid climate. Agric. Water. Manage., 85(1-2), 15-26.

Maestre-Valero, J.F., Martínez-Álvarez, V., González-Real, M.M. (2013). Regionalization of the Hargreaves coefficient to estimate long-term reference evapotranspiration series in SE Spain. Span. J. Agric. Res., 11(4), 1137-1152.

Martí, P. \& Zarzo, M. (2012). Multivariate statistical monitoring of $E T_{0}$. A new approach for estimation in nearby locations using geographical inputs. Agr. Forest. Meteorol., 152(1), 125-134.

Martí, P., Zarzo, M., Vanderlinden, K., Girona, J. (2015). Parametric expressions for the adjusted Hargreaves coefficient in Eastern Spain. J. Hydrol., 529(10), 1713-1724.

Martínez-Cob, A., Tejero Juste, M. (2004). A wind-based qualitative calibration of the Hargreaves $\mathrm{ET}_{0}$ estimation equation in semiarid regions. Agric. Water Manage., 64(3), 251-264.

Mendicino, G., Senatore, A. (2013). Regionalization of the Hargreaves coefficient for the assessment of distributed reference evapotranspiration in Southern Italy. J. Irrig. Drain. Eng., 139(5), 349-362.

Ravazzani, G., Corbari, C., Morella, S., Gianoli, P., Mancini, M. (2012). Modified HargreavesSamani equation for the assessment of reference evapotranspiration in Alpine River Basins. J. Irrig. Drain. Eng., 138(7), 592-599.

Samani, Z. (2000). Estimating solar radiation and evapotranspiration using minimum climatological data. J. Irrig. Drain. Eng., 126(4), 265-267.

Shahidian, R., Serralheiro, P., Serrano, J., Teixeira, J.L. (2013). Parametric calibration of the Hargreaves-Samani equation for use at new locations. Hydrol. Process., 27(4), 605616. 
Thepadia, M. \& Martínez, C.J. (2012). Regional calibration of solar radiation and reference evapotranspiration estimates with minimal data in Florida. J. Irrig. Drain. Eng., 138(2), 111-119.

Vanderlinden, K., Giráldez, J. V., and Van Meirvenne, M. (2004). Assessing reference evapotranspiration by the Hargreaves method in Southern Spain. J. Irrig. Drain. Eng., 130(3), 184-191. 